\section{Über das Vorkommen des trans-Isomeren im natürlichen Blätteralkohol}

\author{
Von A. Hatanaka und M. Ohno \\ Aus dem Institut für chemische Forschung, \\ Universität Kyoto (Japan) \\ (Z. Naturforschg. 15 b, 415 [1960] ; eingegangen am 11. März 1960)
}

Das cis-trans-Problem des natürlichen Blätteralkohols, das von StoLl ${ }^{1}$ und $\mathrm{T}_{\mathrm{AKEI}}{ }^{2}$ seit 1938 bearbeitet wurde, konnte von Crombie ${ }^{3}$ an Hand des IR-Spektrums zugunsten der cis-Konfiguration entschieden werden. Seitdem sind an der Konfiguration des natürlichen Blätteralkohols als 3-cis-Hexen-1-ol keine Zweifel mehr geäußert worden.

Wir konnten bei genauerer Betrachtung des IR-Spektrums von Crombie eine sehr schwache Bande bei 10,3 $\mu$ nicht übersehen. Zur genaueren Ermittlung dieser für eine trans-Doppelbindung charakteristischen Bande isolierten wir natürlichen Blätteralkohol aus frischen Teeblättern. Diese zeigten im IR-Spektrum zwei scharfe Banden bei 10,3 $\mu$ (trans-Doppelbindung) und 11,5 $\mu$ (cis-Doppelbindung) (Abb. 1 a). Zur Untersuchung der Frage, ob die trans-Form als solche in der Pflanze vorkommt oder aber sich bei der Aufarbeitung aus der cisForm bildet, wurden folgende Versuche unternommen :

1. Synthetisches 3-cis-Hexen-1-ol wurde auf dem Ölbad 10 Stdn. auf $130-170^{\circ}$ erhitzt.

2. Synthetisches 3-cis-Hexen-1-ol wurde in Benzol mit 3.5-Dinitrobenzoylchlorid und einigen Tropfen Pyridin versetzt und 3 Stdn. unter Rückfluß gekocht. Anschließend wurde das 3.5-Dinitrobenzoat durch Wasserdampfdestillation mit $40 \% \mathrm{KOH}$-Lösung verseift.

In beiden Fällen zeigte das nach der Behandlung zurückgenommene 3-cis-Hexen-1-ol keine Änderung der Intensitäten im IR-Spektrum und in anderen physikalischen Eigenschaften.

Diese Ergebnisse zeigen, daß auch der natürliche Blätteralkohol unter den Isolierungsbedingungen beständig sein sollte.

Als nächstes stellten wir aus natürlichem Blätteralkohol das 3.5-Dinitrobenzoat her. Durch vielfache systematische Fraktionierung gelang es uns, daraus den 3-trans-Hexen-1-ol-ester rein zu isolieren. Sein IR-Spek- trum stimmte mit dem des synthetischen 3-trans-Hexen1-ol-3.5-dinitrobenzoesäureesters völlig überein. Gegen-

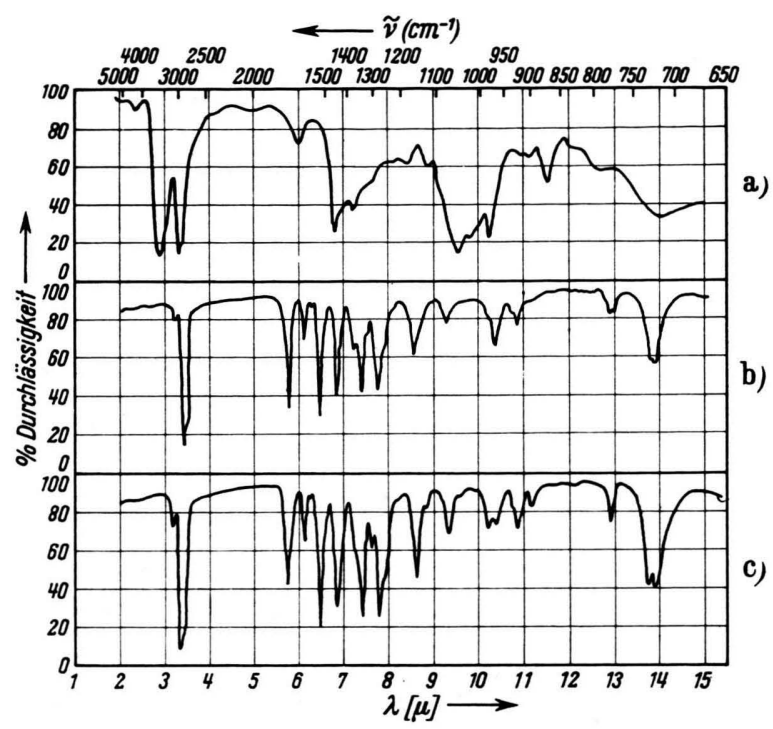

Abb. 1 a. IR-Spektren von natürlichem Blätteralkohol.

Abb. 1 b. IR-Spektren von synthetisiertem 3-trans-Hexen-1-ol-ester.

Abb. 1 c. IR-Spektren von synthetisiertem 3-cis-Hexen-1-ol-ester.

über dem synthetischen cis-Isomeren ergaben sich im IRSpektrum deutliche Unterschiede (Banden bei 10,2 bis $4 \mu, 12,8-9 \mu$, und $13,6-9 \mu$, s. Abb. 1 b, c)

Aus den Fraktionierungs-Versuchen und IR-Spektren geht hervor, daß das 3-trans-Hexen-1-ol zu 3-6\% im natürlichen Blätteralkohol enthalten ist. Demnach besteht der natürliche Blätteralkohol aus Teeblättern aus einem Gemisch der cis-trans-Isomeren, wobei das cisIsomere stark überwiegt.

1 M. Stoll u. A. Rouve, Helv. chim. Acta 21, 1542 [1938]

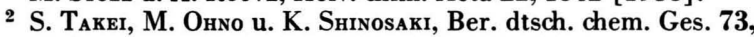
950 [1940].

3 L. Crombie u. S. H. Harper, J. chem. Soc. 1950, 873.

\section{Über die fermentative Reoxydation von Hydrochinon in Kulturhefe}

\section{Von L. KiEsow und U. Schmitz *}

Physiologisch-chemisches Institut der Freien Universität Berlin, Berlin-Dahlem

(Direktor: Professor Dr. Dr. ERnst Schütte)

(Z. Naturforschg. 15 b, 415-416 [1960]; eingegangen am 14. April 1960)

Wenn man zu Kulturhefezellen, die in Phosphatlösung suspendiert sind, z. B. wenig 2-Methyl-1.4-naphthochinon gibt, so wird das Chinon zu seinem Hydrochinon reduziert. $\mathrm{Da}$ gleichzeitig lang anhaltender $\mathrm{O}_{2}$ Verbrauch einsetzt, muß das Hydrochinon in den Zellen wieder reoxydiert werden. Das Chinon geht also eine Katalyse ein, in der die Reduktion fermentativ ist ${ }^{1}$. Daß auch die Reoxydation des Hydrochinons ein fermentativer Vorgang ist, zeigen die Versuche dieser Notiz.

Die Reduktion des Chinons in den Zellen ist ein gegen Blausäure relativ unempfindlicher Vorgang, denn $10^{-2}-m$. KCN hemmen ihn erst zu 50 Prozent. Das gilt aber nicht für die Reoxydation des Hydrochinons. Sie wird durch $1 \cdot 10^{-4}-m$. KCN zu $50 \%$ und durch $1 \cdot 10^{-3}$-m. KCN zu $100 \%$ gehemmt. Als $\mathrm{Maß}$ für die Blausäurewirkung dienen uns die Veränderungen des

\footnotetext{
* Aus der unveröffentlichten Dissertation von U. Schmitz.

1 L. Kiesow, Z. Naturforschg. 15 b, 174 [1960].
} 